\title{
A referential framework for the choice of prototypes in the design phase of a mobile application
}

\author{
Samuel Marie-Rose, Giovanny Arbelaez, Mauricio Camargo \\ Equipe de Recherche sur les Processus Innovatifs, ERPI \\ Université de Lorraine \\ Nancy, France \\ arbelaez5@univ-lorraine.fr
}

\begin{abstract}
This paper aims at providing designers a referential framework for the choice of the most appropriate prototype to use to test a specific aspect of a mobile application during the design phase. In order to reach this objective, a literature review was carried out for characterizing and identifying the different types of prototypes. Four main features were distinguished for characterizing prototypes: level of fidelity, sustainability, medium and interactivity. After this review, a focus was done on the design phase of a mobile application and the referential framework was proposed by defining eight aspects commonly tested while designing. Then, a case study helped to test the framework in order to validate its usefulness and consistency.
\end{abstract}

Keywords - Referential framework, Prototyping, Design phase, Mobile application

\section{INTRODUCTION}

Prototyping has received much attention for many years due to the major role it plays in the design phase of a product [1]. A prototype is an artifact that imitates one or more features of a product, service or system [2]. It is really useful in the design process because it allows having a concrete idea of the final product. However, due to the various types of prototypes that exist, it could be difficult to choose the most appropriate one, to test a specific aspect of a product during its design phase.

One way to help designers to select the most appropriate prototype to use is to define features that allow characterizing the different types of prototypes and there have been various researches regarding that. For example, Baccino [3] proposed to characterize prototypes by using four features: level of fidelity, interactivity, sustainability and medium. Furthermore, more recently [1] provided guidelines for incorporating prototyping in the design process. Nevertheless, although features were defined for prototypes, they did not use it to characterize each one. Moreover, little attention has been paid to the classification/selection of the most appropriate prototype to use for testing a specific aspect of a product during the design phase.

The present paper presents a referential framework for selecting such a prototype. Based on a literature review, it describes the four features that characterize a prototype before providing a set of prototypes usually used. It then proposes a table that characterizes each type of prototype before providing support for selecting the most appropriate prototype to use for testing a specific aspect of a product in the design phase of a mobile application.

\section{LITERATURE REVIEW}

A prototype is defined as an artifact that imitates one or more features of a product, service or system [2]. Prototyping has been key for designing products and solutions. Henry Ford used several prototypes before finalizing the model $\mathrm{T}$ design [4].

This highlights that in addition to being a process included in the design phase of a product/service, prototyping can be used in various fields (manufacturing, marketing, automotive industry or others).

Literature provides much information about when and how to integrate prototyping into the design process. Depending on the desired objective, a specific prototype will be used. During the design phase, it's needed to take numerous factors into account to produce a prototype. Prototypes are assessed on the following features: testing, timing, ideation, feedback and fidelity.

Testing: Although every test includes some cost, effort and time to be produced [5], a separating feature between a prototype and a design concept is the possibility to realize some tests and validate design hypothesis. In a design process, it's important to collect information to move forward in product development without spending a lot of money and time. At this point, prototyping appears to be a response to this need.

Timing: It is widely recognized that the timing of prototyping is important in the design process. Research conducted on prototyping in the early stages of the design phase argued for early and frequent prototyping as a way to test ideas early [6] and to help build confidence in product development in a team [7]. Decisions made in a good timing of a design process have great bearing on the outcome of the design in terms of design success, cost of manufacturing and time to market [8] Ideation: When exploring concepts, prototypes are essential. They enable organic learning and discovery [9] in addition to enabling ideas to be more functional [10]

Feedback: It is important to get feedback at different stages of the design process. This is why prototyping is useful to give to stakeholders, peers and others an idea of the final product and detect possible problems.

Fidelity: The level of fidelity of a prototype must depend on the objective of this prototype. Higher fidelity representations lead to more accurate interpretation, comments and feedback by third parties [11].

While the previously described characteristics are taken into account when defining a prototype, after the literature analysis, it was found that there are mainly four characteristics that are defined before deciding the type of prototype to use when designing a mobile application. Those characteristics are: sustainability, interactivity, level of fidelity and medium. 


\section{A. Sustainability}

A prototype can be throwaway, evolutionary or incremental. Each one has a particular objective.

A throwaway prototype aims at developing a small part of the system and using it to enable end users to try it out and evaluate it [3]. The user provides feedback which can be quickly incorporated into the product development. This cheap and fast prototype is then discarded. An evolutionary prototype is a robust prototype built in a structured manner and constantly refined (c.f. Fig. 1). The artifact, when built, forms the core of the new system and the improvements and further requirements will be then implemented based on the feedback collected [12].

An incremental prototype aims at building and testing progressively the designed product as separate prototypes. At the end, these prototypes are merged in an overall design.

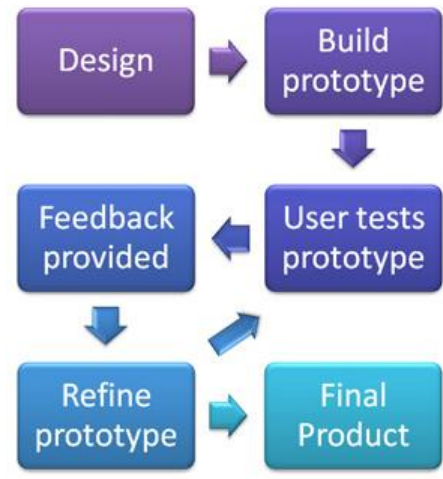

Fig. 1. Steps of an evolutionary prototype

\section{B. Interactivity}

A prototype may be horizontal or vertical.

A horizontal prototype demonstrates only the outer layer of the human interface, such as windows, options and screens. This does not enable direct interaction with the user. This type of prototype is used to check that users understand the structure and functionalities of the product but also to clarify its scope and requirements.

A vertical prototype also called interactive prototype, allows the user to interact with the system [3]. It is an enhanced complete elaboration of a single subsystem or function. This type of prototype is useful for refining database design, collecting information on interface needs and clarifying complex requirements.

\section{Level of fidelity}

A prototype is also characterized by its level of fidelity (c.f. Table 1). The concept of fidelity indicates how closely the prototypes resemble the finished product in terms of interaction, visual appearance, data model and level of detail [13].

Prototypes are usually classified as low-fidelity or high fidelity, but to better describe more recent prototyping approaches, two other classifications has been proposed: mixed-fidelity [14] and multi-fidelity [15].

\section{1) Low-fidelity}

A low-fidelity prototype is sketchy and incomplete. It consists of a series of drafts representing scenarios and flows [16]. It aims at quickly producing the model and broadly testing concepts. This cheap prototyping occurs early in the design process and allow detecting needs of the users and basic usability problems [17].

2) High-fidelity

High-fidelity prototypes are interactive, usually computerbased and present the complete functionality of a service or product. The similarities of this type of prototype and the final product are so high in terms of details and functionality, that users may not note the difference [17]. Furthermore, it is more expensive and time-consuming than low-fidelity prototypes and is usually used at the end of the design process [3].

TABLE 1. ADVANTAGES AND DISADVANTAGES OF LOW-FIDELITY AND HIGH-FIDELITY PROTOTYPES BASED ON [16]

\begin{tabular}{|c|c|c|}
\hline & Advantages & Disadvantages \\
\hline $\begin{array}{l}\text { Low-fidelity } \\
\text { prototypes }\end{array}$ & $\begin{array}{l}\text { - Lower development } \\
\text { cost } \\
\text { - Evaluate multiple } \\
\text { design concepts } \\
\text { - Useful communication } \\
\text { device Address screen } \\
\text { layout issues } \\
\text { - Useful for identifying } \\
\text { market requirements } \\
\text { - Proof-of-concept. }\end{array}$ & $\begin{array}{l}\text { - Limited error checking } \\
\text { - Poor detailed } \\
\text { specification to code to } \\
\text { - Facilitator-driven } \\
\text { - Limited utility after } \\
\text { requirements established } \\
\text { - Limited usefulness for } \\
\text { usability tests } \\
\text { - Navigational and flow } \\
\text { limitations. }\end{array}$ \\
\hline $\begin{array}{l}\text { High-fidelity } \\
\text { prototypes }\end{array}$ & $\begin{array}{l}\text { - Complete functionality } \\
\text { - Fully interactive } \\
\text { - User-driven } \\
\text { - Clearly defines } \\
\text { navigational scheme } \\
\text { - Use for exploration and } \\
\text { test } \\
\text { - Look and feel of final } \\
\text { product } \\
\text { - Serves as a living } \\
\text { specification } \\
\text { - Marketing and sales } \\
\text { tool. }\end{array}$ & $\begin{array}{l}\text { - More expensive to } \\
\text { develop } \\
\text { - Time-consuming to } \\
\text { create } \\
\text { - Inefficient for proof-of- } \\
\text { concept designs } \\
\text { - Not effective for } \\
\text { requirements gathering. }\end{array}$ \\
\hline
\end{tabular}

3) Mixed-fidelity

Mixed-fidelity prototyping combines low-fidelity and highfidelity prototyping in a single prototype. It mixes static layouts and interactive features. It allows designers to focus on a specific interface issue, by exploring and making refinements at higher-fidelity and leaving other elements at lower-fidelity to keep them within the context of the overall design [18]. Its advantages and disadvantages would depend on the chosen combination of the fidelity levels.

\section{Medium}

A prototype can be represented in three different mediums: paper, electronic and video.

\section{1) Paper prototyping}

Paper prototyping is a technique in which paper models are used to create and test user interfaces in an early stage of a design process. It is a quick and cheap way to validate the value of ideas and concepts. Users can have a concrete idea of the main options of the product or service by using these prototypes.

\section{2) Electronic prototyping}

An electronic prototype provides a screen layout that can be static or interactive. It aims at creating a user interface that looks and behaves like the final product. Electronic prototyping can be carried out once the overall flows and 
functionality have been agreed. This iterative design approach is the next step after paper prototyping.

3) Video prototyping

Video prototypes use video to illustrate the user interactions with the system. In this case, the user does not interact directly with the system, but can have a good idea of the system use. It is usually used with a focus group or to complete a paper prototype.

Table 2 presents how appropriate it is to use one specific medium for a prototype regarding the already described characteristics.

TABLE 2. DEGREE OF APPLICABILITY OF THE MEDIUM REGARDING THE TYPE OF PROTOTYPE BASED ON [3]

\begin{tabular}{|l|l|l|l|}
\hline & Paper & Electronic & Video \\
\hline Throwaway & R & R & R \\
\hline Iterative & R & R & NA \\
\hline Incremental & NA & R & NA \\
\hline Horizontal & R & R & NA \\
\hline Vertical & M & R & R \\
\hline Low-fidelity & R & M & M \\
\hline Mixed-fidelity & NA & R & R \\
\hline High-fidelity & NA & R & M \\
\hline
\end{tabular}

NA: Not Applicable; M: Marginal; R: Recommended

\section{Types of prototypes}

While many types of prototypes exist, we found in the literature review ten of the most commonly used:

- Video prototype

- Feasibility prototype developed to determine the feasibility of various solutions. It is used to resolve technical risks related to the development in terms of performance, compatibility of components for instance.

- Vertical prototype

- Horizontal prototype

- Rapid prototyping is a technique of quickly creating a scale model of a part or finished product using a threedimensional computer-aided design

- Simulation prototype is digitally creating a physical product to predict its performance in the real world

- Storyboard is a series of drawings or images that describes a product, service or system in the form of a story. It aims at determining useable sequences for presenting information

- Wireframes are presented in the form of illustrations that capture a specific aspect of design such as an idea, layout or sequence

- Animations are drawn images put in a sequence to present a scenario

- A mock-up provides an overall visual of the product

Depending on what designers are working on, they will select the most appropriate prototype to use. In a design process, when prototyping, it will be useful to determine which specific aspect to test, and after, to choose the prototype that will allow collecting more feedbacks and help to improve the designed product.
The literature review allows having a clear vision of the different types of prototypes existing but also to understand the features that characterizes these prototypes (c.f. Fig. 2).

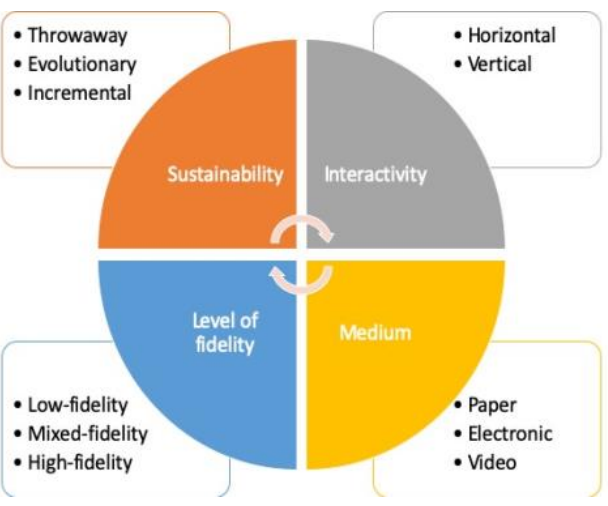

Fig. 2. The four main features of prototypes

Through the review, it is interesting to note that there are links between features. For instance, most of horizontal prototypes are throwaway and are often low-fidelity. We can also remark that most of electronic prototypes are vertical (interactive). Furthermore, based on the literature review, it is interesting to highlight a gap in the characterization of each type of prototype. In fact, at our knowledge, we have no found publications characterizing each prototype and providing information about in which forms the prototype come in. That is why it appears important, in this paper, to propose a table presenting the features of each prototype (c.f. TABLE 3). To achieve this, eight of the ten prototypes presented in the literature review have been selected to be characterized. Based on this review, an analysis aiming at understanding the features of each prototype has been done. TABLE 3 presents the results of this analysis and interpretation.

TABLE 3. CHARACTERIZATION OF EACH PROTOTYPE

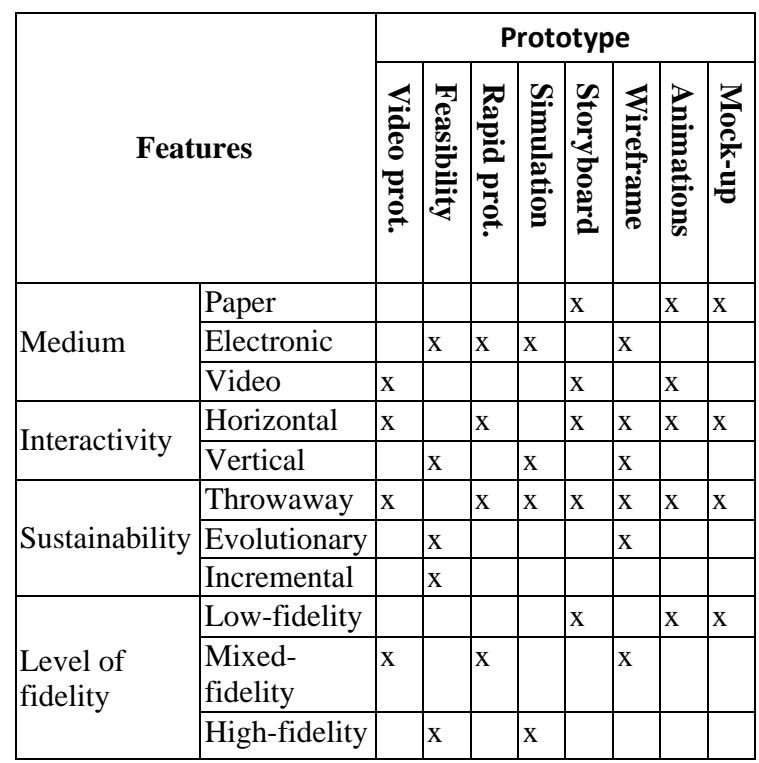

The table can be useful for a designer or a team when choosing a prototype to produce that must involve specific features. For instance, a team which wants to produce an artifact involving the following features: mixed-fidelity, 
throwaway, horizontal (static) and video, can use the table to note that the most appropriate model to produce is the video prototype. Then, this team can easily select this model to realize the desired test.

Another example, by using TABLE 3, a mobile application designer who wants to produce a model involving the following features: high-fidelity, vertical (interactive) and electronic, can note that two types of prototypes seem to be appropriate. These artifacts are the feasibility prototype and simulation prototype. However, these two models are different given that they allow realizing distinct tests. Indeed, while feasibility prototype allows testing technical aspects and compatibility between components for a mobile application, simulation prototype allows testing user interactions with the application. This shows the need to introduce the type of test criteria to help designers in selecting prototype to produce depending on the test to realize. Here is the second contribution of this research aiming at facilitating the choice of the most appropriate prototype to use to test a specific aspect of a mobile application during the design phase.

The present paper focuses its research on mobile applications.

\section{REFERENTIAL FRAMEWORK FOR THE CHOICE OF PROTOTYPES IN THE DESIGN PHASE OF A MOBILE APPLICATION}

In order to support designers in their choice of a prototype for testing a specific aspect of a mobile application, it was critical to distinguish three main development focus of a mobile application in the design phase: the user journey, the valueadded services ideas and the technical challenges. The user journey presents the different layouts and the sequence that will face the customer while using the application. This development focus is critical for allowing customers to interact with the application and for involving them in strategy work [19]. The value-added services ideas of the mobile application are the development focus that aims at determining which services will be developed for adding value to the standard services. When designing the product, it is important to gauge the level of interest of a service idea with the user, to evaluate if it is interesting to develop it. These services depend on the main objective of the application. Finally, the technical challenges refer to the technical obstacles necessary to master in order to implement the mobile application.

With this three-development focus, eight aspects usually tested during the design phase of a mobile application have been distinguished:

- Functional aspect: that aims at imitating the functions of the final product as closely as possible no matter how different they look from the final product. It allows testing the coherence of functionalities proposed and the ease of use of the system.

- Aesthetic aspect: is a factor that plays an important role in user experience. This aspect is closely linked to usability. Users perceive more aesthetic interfaces as much more intuitive than those considered to be less aesthetically pleasing. Indeed, the visual appearance of the application has a positive impact on performance leading to reduced task completion times [20]. The level of aesthetics is mainly determined by colour combination, visual layout and text front of the interface [21]. It is critical to test this aspect to ensure that the interface is easy and pleasant to use.

- Technical feasibility: seeks to explore the technical constraints of complex design features. It allows to study what is feasible or not from a technical and technological point of view (data sharing, data storage or others)

- Interaction: refers to the interaction between the user and the system. It is the ability of the system to respond to a specific command issued by the user

- Interoperability: is the ability of different information technology systems and software applications to exchange data, communicate and use the information that has been exchanged. During interoperability testing, we check how the data from an application/system is transferred into another application/system and further processed to give the accepted output.

- Content: refers to the information presented at each stage of the use of the application

- Usage scenario: refers to the different stages faced by the user to activate and use a service when using the mobile application

- User experience: refers to a person's attitudes and emotions while interacting with a particular system or service [22], [23].

Each aspect is important to test when designing a mobile application in order to measure and assess the level of interest of the user for the product. Moreover, by realizing these tests, designers ensure the design of a consistent and feasible application that matches the needs and expectations of customers. Table 4 is a proposal of a referential framework for the choice of prototypes in the design phase of a mobile application. To use it, the designer must refer to the development focus matter (user journey, value-added services ideas or technical challenges) and after, depending on the aspect to test, he can choose the prototype proposed by the table. In the case of various options, designers can use some factors such as costs or prototyping time to support its choice.

TABLE 4. REFERENTIAL FRAMEWORK FOR THE CHOICE OF PROTOTYPES IN THE DESIGN PHASE OF A MOBILE APPLICATION

\begin{tabular}{|l|l|l|l|}
\hline \multirow{2}{*}{ Aspect to test } & \multicolumn{3}{|l|}{ Development focus } \\
\cline { 2 - 4 } & $\begin{array}{l}\text { User } \\
\text { journey of } \\
\text { mobile } \\
\text { application }\end{array}$ & $\begin{array}{l}\text { Value } \\
\text { added } \\
\text { service } \\
\text { ideas }\end{array}$ & $\begin{array}{l}\text { Technical } \\
\text { challenges }\end{array}$ \\
\hline Functional & Wireframe & NA & NA \\
\hline Aesthetic & Wireframe & NA & NA \\
\hline $\begin{array}{l}\text { Technical } \\
\text { feasibility }\end{array}$ & $\begin{array}{l}\text { Feasibility } \\
\text { prototype }\end{array}$ & $\begin{array}{l}\text { Feasibility } \\
\text { prototype }\end{array}$ & $\begin{array}{l}\text { Feasibility } \\
\text { prototype }\end{array}$ \\
\hline Interaction & $\begin{array}{l}\text { Simulation } \\
\text { prototype }\end{array}$ & NA & $\begin{array}{l}\text { Simulation } \\
\text { prototype }\end{array}$ \\
& Wireframe & & . \\
\hline
\end{tabular}




\begin{tabular}{|l|l|l|l|}
\hline Interoperability & Feasibility & NA & Feasibility \\
\hline Content & Wireframe & NA & NA \\
\hline Usage scenario & Wireframe & Storyboard & \\
& $\begin{array}{l}\text { Video } \\
\text { prototype } \\
\text { prototype }\end{array}$ & $\begin{array}{l}\text { Simulation } \\
\text { prototype } \\
\text { Animation }\end{array}$ & \\
\hline $\begin{array}{l}\text { User } \\
\text { experience }\end{array}$ & Wireframe & $\begin{array}{l}\text { Sideo } \\
\text { prototype }\end{array}$ & NA \\
& Animation & \\
\hline
\end{tabular}

\section{CASE STUDY}

In order to experiment and test the proposed referential framework proposed, we used it in the design phase of a multi-device mobile application realized by a French telecommunications company. This application aims at allowing users to manage their connected objects from their mobile.

The framework was applied on two development focus of the mobile application designed: user journey and value-added services ideas. Indeed, Table 4 was a good support for choosing the most appropriate prototype to use for each test to realize. Furthermore, when choosing, some factors such as costs and prototyping time was important to take into account. TABLE 5 presents the prototypes chosen for each development phase and aspect to test when designing. The following sub-sections provide details about the choices done during the design phase of the mobile application.

\section{A. User journey prototyping}

The first design stages of the application were to develop the management of connected objects inventory and the activation of the telecommunications company services (payments, communication, subscription and content).

The objective was to realize a user journey that allowed customers to add their connected objects on the application, to subscribe to an appropriate offer for each device and to manage these objects. It was also important to develop this journey for value-added services management.

In order to properly design the user journey, it was useful to imagine a concrete case. That is why we imagined the journey for the adding and management of a connected suitcase. Therefore, it was critical to propose various journeys in order to assess and design the adding phase of the suitcase on the application, the payment phase and the stage of management
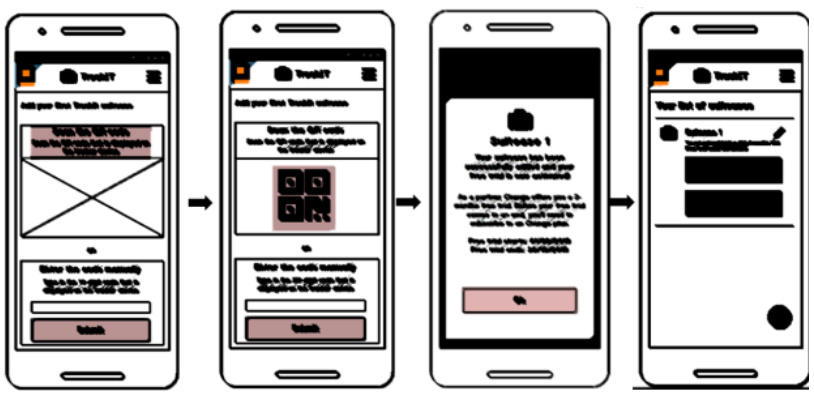

of the connected object. Fig. 3 shows an example of wireframes sequences developed for the user journey.

The aim of this prototype was to test five aspects of the journey: functional, aesthetics, interactivity, content and user experience. Based on the referential framework (c.f. Table 4), wireframes were used with the software Balsamiq for testing the functional, aesthetics, content aspects and the user experience. This prototype allowed visualizing and testing the consistency of the application functionalities, its architecture, the visual quality, the use sequence, the

Fig. 4. Example of storyboard describing an idea to be tested with users

Fig. 3. A sequence of wireframes of the user journey

difficulties that users can face while using the application, the clarity of the content but also the customer behaviour regarding the design of the system.

During this prototyping phase, several iterations were realized in order to improve and enrich the customer journey. Here is one of the advantages of wireframes that allows realizing many iterations before designing a prototype similar to

the final solution and that meets the established requirements. The next steps of the user journey design will aim at testing the technical feasibility of the solutions previously developed.

\section{B. Prototyping of value-added services ideas}

In order to meet the needs of connected objects users and to avoid designing an application that only allows adding and managing devices, it appeared important to identify valueadded services that can allow customers to really take advantage of their connected objects inventory.

To develop and select ideas of services, Design Thinking was used. The first step aimed at identifying and understanding needs, expectations and difficulties of the users regarding the use and management of their connected objects. After, it was critical to define the profile of customers interested in the application before generating value-added services ideas. The following step aimed at prototyping these ideas by producing storyboards to test it with users. Design Thinking method was structured with 5 phases: Empathize - Define - Ideate Prototype - Test.

The storyboards were essential to test the ideas with users. They allowed collecting feedback on the user experience, assessing the coherence of the usage scenario and testing the

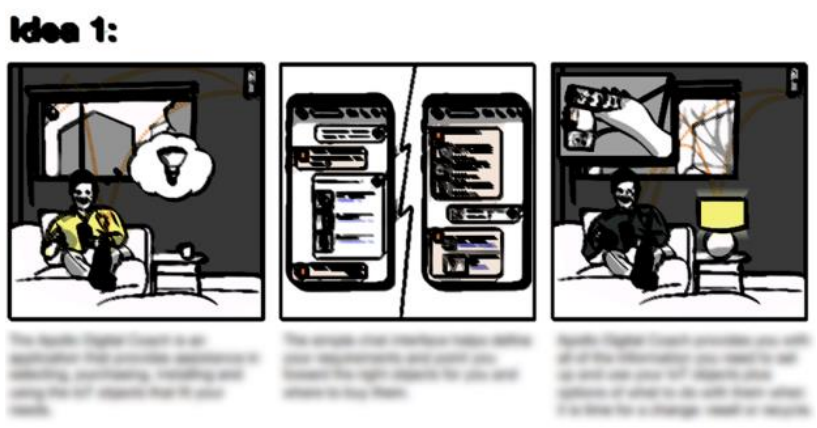

level of interest of the customers for the ideas. Furthermore, 
it is important to note that two factors played a role in the choice of this type of prototype to use. Indeed, the low cost and low prototyping time were factors that encouraged using storyboards besides video prototype and animations (c.f. Table 4).

For the prototyping phase, storyboards were produced to clearly illustrate ideas of services generated during the ideation phase. This type of prototype was appropriate to facilitate the illustration and presentation of the ideas, to assess the user experience regarding these ideas but also to illustrate the usage scenario proposed. Fig. 4 shows a storyboard produced to illustrate an idea and to be tested with users. Each storyboard is composed of three drawings and texts to describe the scenario imagined.

TABLE 5 presents the prototypes used during the design phase of the mobile application based on the referential framework.

TABLE 5. PROTOTYPES USED DURING THE DESIGN PHASE OF THE MOBILE APPLICATION BASED ON THE REFERENTIAL FRAMEWORK PROPOSED

\begin{tabular}{|l|l|l|}
\hline \multirow{2}{*}{ Aspect to test } & Development focus \\
\cline { 2 - 3 } & User journey & $\begin{array}{l}\text { Value added } \\
\text { service ideas }\end{array}$ \\
\hline Functional & Wireframe via Balsamiq & NA \\
\hline Aesthetic & Wireframe via Balsamiq & NA \\
\hline $\begin{array}{l}\text { Technical } \\
\text { feasibility }\end{array}$ & Not tested & Not tested \\
\hline Interaction & $\begin{array}{l}\text { Simulation prototype } \\
\text { (chatbot) }\end{array}$ & NA \\
\hline Interoperability & Not tested & NA \\
\hline Content & Wireframe via Balsamiq & NA \\
\hline Usage scenario & Wireframe via Balsamiq & Storyboard \\
\hline User experience & Wireframe via Balsamiq & Storyboard \\
\hline
\end{tabular}

\section{CONCLUSION AND PERSPECTIVES}

This research aimed at proposing a referential framework for the choice of prototypes in the design phase of a mobile application. Firstly, a literature review provided us with an outlook of the work done in previous research on prototyping and their features. On this review, we distinguished four main features of prototypes: level of fidelity, sustainability, medium and interactivity. In addition to these features, ten types of prototypes commonly used have been identified. A table characterizing each one of these prototypes have been proposed. Furthermore, this allowed us to propose a referential framework for supporting designers in their choice of the most appropriate prototype to use to test a specific aspect of a mobile application during the design phase. Then, a case study was carried out to test this framework and two of three development focus were tested.

In order to go further with this research, it could be interesting to develop and explore three points:

- Although eight types of prototypes were identified for testing different aspects, some other models could be interesting and adapted for testing these aspects. In order to enrich the contribution, we could investigate other prototypes and analyse their benefits and features to determine which aspects they could allow to test
- Furthermore, for proposing the referential framework (c.f. Table 4), eight aspects to test during the design phase of a mobile application were distinguished. However, we could identify more aspect for testing. Depending on the project and the designed application, it could be interesting to test other aspects that could help designers to improve the system. Further research could aim at investigating these aspects.

- Finally, in order to better support design teams, we could propose a detailed list of existing tools available for each type of prototype and related to digital products development (for example, we can use Balsamiq, Axure RP or Sketch to develop wireframes. To go deeper, we could describe each tool, present its advantages and disadvantages and then indicate when it could be interesting to use it.

\section{ACKNOWLEDGMENT}

S.M.R would like to thank Orange Group and the whole team coordinated by Sébastien Bertrand (at the Direction Connected Objects \& Partnership), who made possible the participation to the design of a mobile application.

\section{REFERENCES}

[7] E. M. Gerber, "Prototyping: Facing uncertainty through small wins," in 17 th International Conference on Engineering Design, ICED 09, 2009, pp. 333-342.

[8] A. Häggman, T. Honda, and M. C. Yang, "The Influence of Timing in Exploratory Prototyping and Other Activities in Design Projects," in ASME 2013 International Design Engineering Technical Conferences and Computers and Information in Engineering Conference, 2013, p. V005T06A023.

[9] C. Gill, E. Sanders, and S. Shim, "Prototypes as Inquiry, Visualization and Communication," 2011.

[10] V. K. Viswanathan and J. S. Linsey, "Build to Learn: Effective Strategies to Train Tomorrow's Designers," in 2012 ASEE Annual Conference \& Exposition, 2012, p. 25.273.1-25.273.14. R. Hannah, S. Joshi, and J. D. Summers, "A user study of interpretability of engineering design representations," J. Eng. Des., vol. 23, no. 6, pp. 443-468, Jan. 2012.

[12] E. J. Christie et al., "Prototyping Strategies: Literature Review and Identification of Critical Variables," in 2012 ASEE Annual Conference \& Exposition, 2012.

[13] M. Walker, L. Takayama, and J. A. Landay, "High-Fidelity or Low-Fidelity, Paper or Computer? Choosing Attributes when Testing Web Prototypes," Proc. Hum. Factors Ergon. Soc. Annu. Meet., vol. 46, no. 5, pp. 661-665, Jan. 2002.

[14] M. McCurdy, C. Connors, G. Pyrzak, B. Kanefsky, and A. Vera, "Breaking the Fidelity Barrier: An Examination of Our Current Characterization of Prototypes and an Example of a Mixedfidelity Success," 2006, pp. 1233-1242. 

Prototyping of User Interfaces," 2007, pp. 150-164.

[16] J. Rudd, K. Stern, and S. Isensee, "Low vs. High-fidelity Prototyping Debate," interactions, vol. 3, no. 1, pp. 76-85, Jan. 1996.

[17] T. Nissinen, "User experience prototyping : a literature review," University of Oulu, Oulu, 2015.

[18] J. Petrie, "Mixed-fidelity prototyping of user interfaces," University of Saskatchewan, 2006.

[19] A. Folstad, "Living Labs for innovation and development of information and communication technology: a literature review," Electron. J. Virtual Organ. Networks, vol. 10, pp. 99-131, 2008.

[20] A. Sonderegger and J. Sauer, "The influence of design aesthetics in usability testing: Effects on user performance and perceived usability,” Appl. Ergon., vol. 41, no. 3, pp. 403-410, Jan. 2010.

[21] S. Lee and R. J. Koubek, "The Impact of Cognitive Style on User Preference Based on Usability and Aesthetics for ComputerBased Systems," Int. J. Human-Computer Interact., vol. 27, no. 11, pp. 1083-1114, Jan. 2011.

[22] M. Hassenzahl, "Hedonic, Emotional, and Experiential Perspectives on Product Quality," Encycl. Hum. Comput. Interact., pp. 266-272, Jan. 2006.

[23] M. Hassenzahl and N. Tractinsky, "User experience - a research agenda," Behav. Inf. Technol., vol. 25, no. 2, pp. 91-97, 2006. 